\title{
Vom Naturalismus zur Nervenkunst. Bemerkungen zur frühen literarischen Produktion Ludwig von
}

\section{Fickers}

Du naturalisme à l'esthétique des nerfs. Quelques remarques sur la production littéraire du jeune Ludwig von Ficker

From Naturalism to "Nervenkunst". Notes on the early literary production of Ludwig von Ficker

\section{Markus Ender}

\section{(2) OpenEdition}

Journals

Édition électronique

URL : http://journals.openedition.org/austriaca/612

DOI : 10.4000/austriaca.612

ISSN : 2729-0603

Éditeur

Presses universitaires de Rouen et du Havre

Édition imprimée

Date de publication : 1 juin 2018

Pagination : 167-184

ISBN : 979-10-240-1233-9

ISSN : 0396-4590

\section{Référence électronique}

Markus Ender, „Vom Naturalismus zur Nervenkunst. Bemerkungen zur frühen literarischen Produktion Ludwig von Fickers", Austriaca [Online], 86 | 2018, Online erschienen am: 01 Juli 2020, abgerufen am 28 Januar 2021. URL: http://journals.openedition.org/austriaca/612 ; DOI: https://doi.org/10.4000/ austriaca.612 


\title{
Vom Naturalismus zur Nervenkunst
}

\author{
Bemerkungen ZUR FRÜHen LITERARISCHEN ProdUKTION \\ LUDWIG VON FICKERS
}

\section{Vorbemerkung}

Der Versuch, den Epochenbegriff des literarischen Naturalismus mit dem Kulturvermittler Ludwig von Ficker (1880-1967) und seiner zwischen 1910 und 1954 erschienenen österreichischen Kunst- und Kulturzeitschrift Der Brenner in Verbindung zu bringen, verspricht - bei oberflächlicher Betrachtung - kein befriedigendes Ergebnis zu zeitigen. Zunächst wird lediglich offenbar, dass der Brenner in der Literaturgeschichtsschreibung oftmals nur eine Randnotiz darstellt, denn die Zeitschrift wird zwar immer wieder angeführt, der Großteil der Artikel beschränkt sich aber auf den Aspekt, dass sie - zumindest in den Jahren vor dem Ersten Weltkrieg - von einer „Mischung von wertkonservativer [...] Orientierung und jedenfalls partieller Aufgeschlossenheit gegenüber der expressionistischen Moderne "1 geprägt war, später dann auf christlich-philosophische und nach dem Zweiten Weltkrieg ausschließlich auf katholische Fragestellungen ausgerichtet war. Die literarische Sozialisation und die ersten schriftstellerischen Versuche des BrennerHerausgebers bleiben demgegenüber meist unerwähnt ${ }^{2}$ bzw. werden

1. Sieglinde Klettenhammer, „,Der Scirocco ist kein Tiroler Kind und was uns im Brenner vorgesetzt, ist alles eher als Tiroler Art'. Die Zeitschrift ,Der Brenner 1910-1915", in Klaus Amann [u.a.] (Hg.): Expressionismus in Österreich, Wien [u.a.], Böhlau, 1994, S. 287-308, hier S. 296.

2. Vgl. exemplarisch die Darstellung in Herbert Zeman (Hg.), Literaturgeschichte Österreichs von den Anfängen im Mittelalter bis zur Gegenwart, Freiburg i.Br. [u. a.], Rombach Verlag ${ }^{2} 2014$, S. 523 f. 
zumeist nur als kurze Zusatzinformation angeführt. ${ }^{3} \mathrm{Zu}$ einem wesentlichen Teil sind diese offensichtlichen Lücken auf den Herausgeber selbst zurückzuführen, denn Ficker hielt sich zeitlebens bewusst im Hintergrund, was seine Arbeit rund um den Brenner betraf und gab sich bedeckt, wenn die Rede auf seine frühe literarische Betätigung fiel. In diesem Licht besehen, erscheint kaum verwunderlich, wenn in einem Nachruf aus dem Jahr 1967 folgende Zeilen zu lesen sind: „Er sprach nie über die Gedichte und dramatischen Versuche seiner Jugend, und er sprach nur lächelnd über sein Mißverhalten zur Wissenschaft, zur Universitas." 4

Ludwig von Ficker hatte, im Gegensatz zu anderen Vermittlerpersönlichkeiten wie z.B. Herwarth Walden im Sturm, keine theoretischen Schriften verfasst, die über sein Kunstverständnis hätten Auskunft geben können und es nachvollziehbar gemacht hätten. Die Annäherung an die Frage, welche Bedeutung Ludwig von Fickers Jugenddichtungen beigemessen werden kann und inwieweit in seiner Arbeit vor der Gründung des Brenner Schnittpunkte mit dem literarischen Naturalismus bzw. der Moderne existieren, muss somit über Umwege erfolgen und das kulturelle Dispositiv Tirols um die Jahrhundertwende, die produktive Dimension (d.h. Ficker als Autorsubjekt) wie auch die Rezeption im Blick behalten. Die umfangreiche Korrespondenz Fickers, die im Innsbrucker Brenner-Archiv verwahrt wird, dient in diesem Fall als breite Quellenbasis für einen synchronen Querschnitt ${ }^{5}$; daneben legt die Spurensuche im Umfeld von Fickers eigentlichem Lebenswerk, dem Brenner, in diachroner Sicht bestimmte Entwicklungslinien offen.

Bezogen auf sein Geburtsjahr, trat Ludwig von Ficker zu einem Zeitpunkt in seine aktive Schaffensphase ein, als gängige Literaturgeschichten den Naturalismus bereits als im Abklingen begriffen darstellen. ${ }^{6}$

3. Vgl. den Beitrag in Walther Killy (Hg.), Literaturlexikon. Autoren und Werke in deutscher Sprache, Bd. 3, München, Bertelsmann 1989, S. 375.

4. „Ludwig von Ficker zum Gedenken. Ein Prisma unendlicher Reinheit zerbrach“, Tiroler Nachrichten, 22.03.1967, S. 5 .

5. Der mehr als 17.000 Korrespondenzstücke umfassende Gesamtbriefwechsel wird derzeit im Zuge eines FWF-Forschungsprojektes am Brenner-Archiv in eine digitale Edition überführt (FWF-Projekt „Ludwig von Ficker: Digitale Edition und Monografie" [P 29070-G23]; Leitung: Univ.-Prof. Dr. Ulrike Tanzer). Ein Teil der nachfolgend zitierten Briefe stellt bisher unveröffentlichtes Material dar und speist sich aus diesem Datenbestand.

6. Vgl. etwa Günther Mahal, Naturalismus, München, Wilhelm Fink Verlag, 1975, S. 23; Mahal setzt die Phase des „Hochnaturalismus" beispielsweise in der Zeit von 1890-1895 an. 
Doch die Annahme, die komplexe literarhistorische Situation die mit dem Terminus „Naturalismus" umrissen wird, wäre im Falle der Kunstproduktion des jungen Dichters nicht von Bedeutung gewesen, greift mit der einfachen Formel von der späten Geburt zu kurz. Der Grad der Beeinflussung ist hier allerdings mindestens ebenso schwierig zu bestimmen wie das Unterfangen, eine Form des Naturalismus österreichischer Prägung zu umreißen. In dieser Frage erscheint zunächst zweckmäßig, davon auszugehen, den Naturalismus als ,eigenständige Diskursformation innerhalb eines größeren literaturgeschichtlichen Epochenzusammenhangs ${ }^{\text {" } 7}$ zu interpretieren, wobei der Aspekt der zeitgenössischen „unklaren Selbstverortung, die den Naturalismus zwischen bewusster Anknüpfung an das Bestehende und radikalem Bruch mit der Tradition oszillieren lässt" ${ }^{\text {" }}$, auch für die Situation in Österreich gilt. Das muss aus diskursanalytischer Sicht keinen Nachteil darstellen; die Ambivalenz macht vielmehr deutlich, wo die Ränder des Diskurses verlaufen, wenn die theoretisch-normativen Fundierungen der Epoche, wie sie beispielsweise Maximilian Harden dargelegt hat ${ }^{9}$, als Richtgrößen dienen.

\section{Einflussbereiche: Tirol, München, Mähren}

Ludwig von Ficker wurde 1880 als unehelicher Sohn des renommierten Historikers Julius von Ficker und der aus Bruneck stammenden Lehrerin Maria Tschafeller in München geboren und verlebte auch seine Kindheit in dieser Stadt. Erst 1886 übersiedelte die Familie nach Innsbruck, was für den jungen Ludwig einen „schmerzlichen Einschnitt“10 bedeutete. Der Berufswunsch des Jungen bestand darin, Schauspieler

7. Wolfgang Bunzel, Einführung in die Literatur des Naturalismus, Darmstadt, WBG, 22011, S. 7.

8. Ebd., S. 10.

9. „Was will der Naturalismus? Er fordert Abwendung von aller Konvention, Umkehr zur rücksichtslosesten Wahrheit ohne jedes Kompromiß, [...] um den Menschen als Resultat seiner Lebensbedingungen und Umgebung, nicht als Zufallsprodukt einer schönheitsdurstigen Phantasie erscheinen zu lassen." Zit. nach Maximilian Harden, „Die Wahrheit auf der Bühne“, Der Kunstwart. Rundschau über alle Gebiete des Schönen, Jahrg. 1, 15. Stück, 1887, S. 202-205; hier S. 202.

10. Anton Unterkircher, „Ludwig von Ficker“, in Zeitmesser. 100 Jahre „Brenner“, hg. vom Forschungsinstitut Brenner-Archiv der Universität Innsbruck, Innsbruck, iup, 2010, S. 31-55; hier S. 33. 
und Literat zu werden, ${ }^{11}$ und er verfolgte dieses Ziel mit entsprechendem Eifer, doch die eigenwilligen Vorstellungen des Sohnes zogen den Unmut seines Vaters auf sich. Dieser hatte für Ludwig das Studium der Rechtswissenschaften vorgesehen und versuchte deshalb, die künstlerischen Ambitionen seines Sohnes zu unterbinden, was ihm allerdings nicht gelang.

Um die Jahrhundertwende hatte sich Ficker schließlich in Tirol akkommodiert und begann, sich auf dem kulturellen Feld zu orientieren. Er trat dem Akademischen Gesangsverein „Skalden“ bei, baute sich mit gleichgesinnten, künstlerisch orientierten Schulkameraden ein Netzwerk auf und schloss sich der "Jung-Tirol“-Bewegung an, die sich als Gegenkraft zu den reaktionär-konservativen Alt-Tirolern verstand, die dem Ultramontanismus anhingen und sich katholisch, reaktionär und habsburgtreu gebärdeten. Entsprechend gestaltete sich die ideologische Basis der Jung-Tiroler deutschnational und, unter dem Schlagwort „Los von Rom", anti-klerikal. ${ }^{12}$ Ficker bewegte sich in dieser Lebensphase unter anderem im Umfeld von Hugo Greinz, des Herausgebers der in Linz erscheinenden, deutschnationalen Zeitschrift Der Kyffhäuser (1899-1902). Greinz hatte in der ersten Nummer des Kyffhäuser in einer kurzen, programmatischen Abhandlung mit dem Titel „Provinzliteratur" die normative Richtung der „neuen“ literarischen Expression vorgegeben und war entschieden für eine Form regionaler Literaturproduktion eingetreten. Er reagierte damit auf Peter Rosegger und insbesondere auf Hermann Bahrs „Entdeckung der Provinz ${ }^{\text {“ } 13}$ und postulierte:

Von der Provinzliteratur verlangen wir also ganz bestimmte Darstellungen, sie soll uns Charaktere zeichnen, die in den vielen Einflüssen provinzieller Umgebung entstanden und aufgewachsen sind, sie soll uns die Stimmung

11. Von diesen Bestrebungen zeugen noch einige Bildpostkarten, auf denen Ficker in verschiedenen Rollen abgebildet ist, sowie Programmzettel von Aufführungen und Inszenierungen, die im Archivbestand überliefert sind. Vgl. Brenner-Archiv (im Folgenden kurz: BA), Nachlass Ludwig von Ficker, Sign. 95/38-1.

12. Die künstlerische Agitation des jungen Dichters kann auf biographischer Ebene als Schritt in einem emanzipatorischen Prozess wahrgenommen werden, dem ein tief gehender Vater-Sohn-Konflikt vorausging; dieser Prozess lässt sich auf einer allgemein-diskursiven Ebene mit der (zumindest theoretisch) revolutionären Intention von „Jung-Tirol“ zur Deckung bringen.

13. Hermann Bahr, „Die Entdeckung der Provinz“, Neues Wiener Tagblatt, Nr. 270, 01.10.1899, S. 1-3. 
geben, die an ein bestimmtes Land, an eine bestimmte Stadt gebunden ist, - ihre Werke sollen Provinzluft athmen! ${ }^{14}$

Es ist davon auszugehen, dass der junge Ficker diese theoretischen Positionen kannte. Bestimmte formale wie inhaltliche Aspekte seiner Dichtung sprechen dafür, dass ihm daran gelegen war, die Vorgaben praktisch in seinen beiden Dramen Sündenkinder (1900) und Und Friede den Menschen! Eine Christnachtstragödie (1901) umzusetzen. Allerdings illustrieren seine Werke auch, dass er keine unbedingte Hinwendung an das Programm im Sinne der ausschließlichen Fokussierung auf die "provinzielle Umgebung“ des Tiroler Raums praktizierte, sondern vielmehr eine ambivalente Adaption bestimmter Elemente betrieb. Der im unmittelbaren Nahbereich Fickers gelebte Regionalismus und der Deutschnationalismus der "Jung-Tiroler" waren hingegen aber nur von geringer Wirkung auf den jungen Autor. Viel eher zeigte er sich durch arrivierte Dichterpersönlichkeiten des (deutschen) Naturalismus beeinflusst, wobei hier ein mehrere Jahre andauernder Kulturtransfer zwischen München und Innsbruck als ausschlaggebendes Moment zu sehen ist.

Dass Ficker über den provinziellen Tellerrand blickte und neben Gerhart Hauptmann, den die "Jung-Tiroler" bald als „den hervorragendsten deutschen Dramatiker"15 vereinnahmten, beispielsweise auch die Werke von Hermann Sudermann oder Max Halbe rezipierte, war in der Hauptsache dem Umstand geschuldet, dass er es verstand, trotz der Übersiedelung nach Innsbruck engen brieflichen und persönlichen Kontakt zu seinem früheren Bekanntenkreis in München zu halten. Durch die Korrespondenz blieb der Zugang zum kulturellen Leben der Großstadt aufrecht und es war ihm auf diese Weise möglich, die „Brückenfunktion Münchens zu den deutschsprachigen Kulturräumen in der Mitte und im Südosten Zentraleuropas " ${ }^{\text {"16 }}$ zu nutzen und Einflüsse $\mathrm{zu}$ antizipieren, die in Tirol aufgrund des engen Korridors, den die „Provinzliteratur" vorgab, kaum zum Tragen kommen konnten bzw. von anderen regionalen Dichtern von vornherein nicht aufgegriffen wurden.

Im Briefbestand des Nachlasses existieren einige Belege für den Stellenwert, den Hauptmann um die Jahrhundertwende innerhalb dieser

14. Hugo Greinz, „Provinzliteratur“, Der Kyffhäuser. Deutsche Monatshefte für Kunst und Leben, Erster Jahrgang 1899, 1. Heft, S. 16-17; hier S. 17.

15. Tiroler Anzeiger, 16.01.1900.

16. Bunzel, Einführung in die Literatur des Naturalismus, a.a.O., S. 47. 
transnationalen Gruppierung von „jungen Wilden“ einnahm. Hauptmann war im sozialen Umfeld Fickers in München gewissermaßen zum künstlerischen Orientierungsmaßstab erhoben worden, wie sein Freund Carl Spitzweg brieflich am 30. November 1898 mitteilte:

Ganz besonders vorzügliche Darstellung erfahren die Werke Hauptmanns; dazu wöchentlich fast eine Premiere, das ist viel und wird hier nirgend sonst geboten. Man sieht förmlich wie fortschreitend Stein auf Stein gelegt wird zu einem schönen Gebäude ... wer mitbauen konnte ... hin!! ${ }^{17}$

Auch Heinrich Stammberger, ebenfalls ein ehemaliger Schulfreund, empfahl die Dramen Hauptmanns direkt an Ficker weiter, wie er auch den Einfluss des Dichters auf seine Person nicht verleugnen konnte: „Soeben aus Gerhard [sic] Hauptmann ,Vor Sonnenaufgang' (schön) gekommen, sitzen wir im Sommergarten und senden herzliche Grüße an Dich u. Deine lieben Angehörigen [...]. Das Stück enthält viel Wasser auf meine Mühle - und beinah auch für meine Augen. "18 Die Vermittlungsbestrebungen aus der Großstadt sind in Innsbruck nicht ohne Echo geblieben; Ficker übernahm die Empfehlungen und trachtete, sie in seinem neuen Umfeld auch umzusetzen, indem er, im Rahmen seiner Möglichkeiten, private Aufführungen von Hauptmanns Dramen organisierte.

Ludwig von Ficker kann aus diesem Grund als ein Bindeglied zwischen der „modernen“, großstädtischen Atmosphäre eines der naturalistischen Zentren Deutschlands und dem eher ländlich geprägten Milieu der Kleinstadt in Tirol wahrgenommen werden. In der kunsttheoretischen Offenheit, die als Effekt dieses Kulturtransfer festzustellen ist, liegt auch der Schlüssel für die spätere Arbeitsweise Fickers begründet, bei der er sich beispielsweise aktiv gegen „Jung-Tirol“ und die „Skalden“ stellte, als er nach dem Ersten Weltkrieg entschieden für Karl Kraus eintrat. ${ }^{19}$

Ein weiterer direkter Bezug zur Kulturszene Münchens bestand in der Person des Schriftstellers Kurt Martens. Ficker hatte bereits Ende 1899

17. Brief Carl Spitzweg an Ludwig von Ficker, 30.11.1898. BA, Nachlass Ludwig von Ficker, Sign. 42/7-3.

18. Brief Heinrich Stammberger an Ludwig von Ficker, 16.04.1898. BA, Nachlass Ludwig von Ficker, Sign. 46/31-2.

19. Als es 1920 im Zuge einer Kraus-Lesung in Innsbruck zu antisemitischen Ausfällen kam, sah sich Ficker veranlasst, aus der Sängerschaft auszutreten und publizistisch gegen die Rädelsführer vorzugehen; vgl. Sigurd Paul Scheichl, „Aspekte des Judentums im ,Brenner' (1910-1937)“, in Walter Methlagl, Eberhard Sauermann, Sigurd Paul Scheichl (Hg.), Untersuchungen zum,Brenner'. Festschrift für Ignaz Zangerle zum 75. Geburtstag, Salzburg, Otto Müller Verlag, 1981, S. 70-121. 
ein Exemplar seines Dramas Sündenkinder an Martens übersandt, wofür dieser sich brieflich bedankte und mit wohlwollenden Worten insbesondere „die dramatische Steigerung und die Führung des Dialoges“ hervorhob, die ihm "trefflich gelungen“ 20 erschien. Martens' Urteil musste für Ficker von einiger Bedeutung gewesen sein, denn er übertrug diese Briefpassage handschriftlich auf die Rückseite des Zeitungsausschnittes mit einer Rezension zu den Sündenkindern, die er den Innsbrucker Nachrichten vom 24. Dezember 1899 entnommen hatte. ${ }^{21}$

Im Februar 1902 ließ Ficker sein zweites Drama Und Friede den Menschen! durch die Österreichische Verlagsanstalt an Martens senden, und abermals folgte ein Dankesschreiben. Die Gegenbriefe Fickers sind leider nicht erhalten geblieben, die Ausführungen Martens' lassen jedoch indirekt darauf schließen, dass Ficker in einem Begleitschreiben am Beispiel seines Stückes allgemein zu seinem Dichtungsverständnis Stellung bezogen und dabei einen Kernbegriff des literarischen Naturalismus ins Treffen geführt haben musste. Martens schrieb:

Ich habe es [= das Drama, Anm.] mit großem Interesse gelesen und stimme mit den verschiedenen Kritiken, die ich darüber las und die es durchweg rühmten, durchaus überein - das Wesen des Dramatischen fasse ich vielleicht nicht ganz so auf wie Sie. Ich suche es weniger im naturalistischen Milieu als in der rapiden Entwicklung von Entschlüssen und Handlungen; dagegen bin ich voll Bewunderung für die Treue und Echtheit Ihrer Charaktere und Situationen und besonders für Ihren knappen, verinnerlichten Dialog. ${ }^{22}$

Neben den Einflüssen aus Tirol und München fand Ludwig von Ficker seinen wichtigsten Impulsgeber in der Person Franz Schamanns (1876-1909), eines mährischen Schriftstellers, mit dem er zwischen 1900 und 1903 in regem brieflichen Kontakt stand und auch mehrmals persönlich zusammentraf. Schamann wurde für Ficker in dieser Zeit zum Maßstab des Künstlers, der sein Künstlertum gegen alle Widrigkeiten des Lebens durchzusetzen musste. Er war ein schwieriger Charakter voller Widersprüche; in seinem Lebenswandel schien er die Programmatik des Naturalismus verinnerlicht zu haben (durch permanente Armut, Geschlechtskrankheit, übersteigerte Triebhaftigkeit und den Glauben

20. Brief Kurt Martens an Ludwig von Ficker, 26.12.1899. BA, Nachlass Ludwig von Ficker, Sign. 30/46-1.

21. Vgl. Innsbrucker Nachrichten, 24.12.1899. BA, Allgemeine Zeitungsausschnittesammlung.

22. Brief Kurt Martens an Ludwig von Ficker, 14.02.1902. BA, Nachlass Ludwig von Ficker, Sign. 30/46-2, Hervorhebung d. Verf. 
an eine Form des Determinismus) ${ }^{23}$. Entsprechend kümmerte er sich wenig um Konventionen bzw. literarische Theorien. Dennoch setzte sich Ficker, der, wie der Briefwechsel illustriert, von der Persönlichkeit seines Freundes fasziniert war, näher mit seiner Literatur auseinander (wie es umgekehrt auch Schamann tat).

Auch Schamann richtete seine künstlerische Produktion am Vorbild der ausländischen Naturalisten aus. So gab er an, dass sein unveröffentlicht gebliebenes Drama „Elend“ 24 unmittelbar auf der HauptmannLektüre gründet: „[...] alle meine Dramen endigen so, wie Sie sich an dem, mit gleicher Post an Sie abgehenden Stücke ,Elend' (,Die Ziegler') überzeugen können. Dieses Stück habe ich nach Lesung der Hauptmann'schen "Weber" mit 17 Jahren geschrieben. ${ }^{25}$ Die wichtigste Inspirationsquelle stellte für Schamann aber Émile Zola dar, wie er in einer ausführlichen Briefpassage betonte:

Zola ist tot! Ein edles Tier ist in der Wüste gefallen - da sind auch schon die Aasgeier da. Was ist über den Toten geschrieben worden? Jeder Schmock musste seiner Dummheit tintnen Ausdruck geben. Und da die Schmöcke schrieben ... darum wollen wir schweigen. Was können wir uns über unsern Lehrer sagen? Dass er ein Gigant war, vielmehr ist!, der uns mit seinen kräftigen Armen emporhob, dass es uns verflucht schmerzte, emporgestellt hat auf seine Höhe, sodass wir die Leute mit seinen Augen zu schauen uns angewöhnten? Dass wir ihm dafür nicht danken dürfen, sondern ihn stets ehren müssen? Dass er nicht sterben kann, bis die Freiheit wieder da ist, nach der er sich als Daseiender vergeblich gesehnt hat? - Sollen wir beide uns dies Alles in langer Rede sagen? Zola Lehrer!! ${ }^{26}$

Bestätigt dieses unbedingte Bekenntnis zu Zola als dem „Lehrer“ die Hinwendung Schamanns zum literarischen Naturalismus, so scheint er in späteren Briefen an Ficker paradoxerweise eben dieses Bekenntnis wieder zu negieren. Wenige Monate nach dem Tod Zolas brach Schamann, seinem impulsiven Wesen entsprechend, mit den formalen Determinanten, die in der naturalistischen Theorie eingefordert worden

23. Vgl. Reinhild Kaufmann, Franz Schamann. Eine Monographie, Univ.-Diss., Innsbruck, 1974.

24. Typoskript „Elend (Die Ziegler)“(Drama, 1901). BA, Nachlass Ludwig von Ficker, Sign. 74/21-2.

25. Brief Franz Schamann an Ludwig von Ficker, 28.02.1902. BA, Nachlass Ludwig von Ficker, Sign. 42/48-5.

26. Brief Franz Schamann an Ludwig von Ficker, 02.10.1902. BA, Nachlass Ludwig von Ficker, Sign. 42/47-1. 
waren und beschrieb in einem Brief an Ficker im Jänner 1903 seine geänderte Haltung gegenüber den Naturalisten wie folgt:

Ich halte mich, wie Sie aus allen meinen Dramen ersehen können, nicht an die momentan herrschende Schule der modernen Dramatiker. Dieser Leute Tun ist mir ganz egal. Sie sollen meinetwegen der Echtheit zuliebe sogar wirkliche Aborte samt deren Inhalt auf die Bühne stellen; die Leute werden ,Bravo! Gott, wie natürlich!' rufen - das tangirt mich nicht. Sie mögen die Figuren den unverfälschtesten, geistlosesten Dialekt sprechen lassen, (Zusatz: siehe oben) - das tangirt mich nicht. (Ich könnte Ihnen noch einige Regeln der Herren Naturalisten anführen, die mich ganz kalt lassen!) Wenn meine Gestalten etwas nachdenken und ihrem Denken Wort verleihen, so bezwecke ich damit nichts andres, als dass der Zuhörer mitdenken soll; dafür kommt er ja ins Theater! ${ }^{27}$

Es ist davon auszugehen, dass Schamann hier Ficker gegenüber eine Form der Selbstinszenierung betrieben hat, indem er versuchte, sich über den theoretischen Kosmos derer zu heben, an deren Positionen er sich noch kurz zuvor orientiert hatte. Unabhängig davon thematisierte er aber auch einen Problembereich, an dem der Naturalismus laborierte, denn das formale Repertoire, das für den literarischen Ausdruck zur Verfügung stand, war durch die materialistische Fokussierung notwendigerweise beschränkt.

Jene Aspekte, die Ficker an Schamanns Dichtung schätzte, zeichnen sich durch die Differenz aus, mit denen der Naturalismus vom poetischen Realismus abgegrenzt wurde (und nach wie vor wird). In besonderem Maße gilt das für die Schilderung des Milieus, denn Schamann, so Ficker in einer Rezension :

zeichnet das Leben seiner Mährischen Landsleute, so weit es in den Bereich seiner Darstellung fällt, nicht, wie es sein soll - nein, wie es ist: brutal und beutelüstern, oft nur rein thierischen Instincten aufgeopfert, mit einem starken sexuellen Einschlag. [...] Bei Schamann ist alles in Aufruhr und Bewegung. Seinen Novellen ist durchwegs ein revolutionärer Charakter imprägnirt, sei es in socialer Hinsicht oder auf dem Gebiete der Moral. ${ }^{28}$

Ficker apostrophierte insbesondere die deutlichen Referenzen, die Schamanns Literatur zum russischen Naturalismus und damit zum sozialrevolutionären Impetus aufweist, indem er Parallelen zur Dichtung Maxim Gorkis zog.

27. Brief Franz Schamann an Ludwig von Ficker, 24.01.1903. BA, Nachlass Ludwig von Ficker, Sign. 43/1-1.

28. Ludwig von Ficker, „Franz Schamann: Mährische Geschichten “ [Rezension], Tiroler Anzeiger, Nr. 112/1902, S. 4. 
All diese Einflussfaktoren fanden in der Literaturproduktion Ludwig von Fickers ihren Niederschlag, und ebenso heterogen wie die Präliminarien gestalteten sich Gehalt und Gestalt seiner eigenen Werke. Ficker hat nur wenig Literarisches veröffentlicht, es existieren lediglich zwei Dramen und ein schmales Gedichtbändchen, ${ }^{29}$ daneben fanden einige Gedichte bzw. kurze Prosaskizzen ihren Weg in diverse Zeitungen. Die im Brenner-Archiv überlieferten literarischen Arbeiten aus den Jahren 1897-1909 bestehen in der Hauptsache aus einer erklecklichen Anzahl von Entwürfen, Skizzenblättern und -büchern, ${ }^{30}$ die der Brenner-Herausgeber über annähernd sieben Jahrzehnte bis zu seinem Tod aufbewahrt hatte. Der größte Teil ist unveröffentlicht geblieben, die Entwürfe sind zudem vielfach nur unvollständig überliefert bzw. wurden von Ficker überhaupt nur fragmentarisch ausgeführt. Allerdings können bereits anhand der Titel Rückschlüsse auf den diskursiven Rahmen, in dem sie entstanden sind, gezogen werden. So sind im Falle der Titelei der Skizze „Nach Sonnenuntergang “31 sowie der Novelle „Bahnwärters Christnacht ${ }^{\text {“32 }}$ Bezugnahmen auf Texte Hauptmanns („Vor Sonnenaufgang“ bzw. „Bahnwärter Thiel“) als wahrscheinlich anzunehmen.

In den Werken Fickers aus den Jahren zwischen 1897 und 1909 begegnet man, insbesondere in seinen Dramen, im Sinne des eingangs zitierten Oszillierens zwischen Tradition und Neuerung, sowohl „klassischen“ Elementen als auch solchen der "modernen“ Provinzkunst (die hier eher in einer lockeren Adaption der Greinz'schen Positionen besteht und nicht mit der reaktionär ausgerichteten „Heimatkunst“33 gleichzusetzen ist), sowie Versatzstücken des literarischen Naturalismus, dessen theoretische Fundierung dem jungen Ficker durchaus bekannt war. Er gesellte sich damit in die Reihe jener Dichter, die um die Jahrhundertwende vermittelst der „Ankopplung an die gesellschaftliche

29. Inbrunst des Sturms. Ein Reigen Verse, von Ludwig von Ficker, Leipzig-Berlin, Modernes Verlagsbureau Curt Wigand, 1904.

30. Vgl. Ludwig von Ficker, „Schlagschatten. Ein Skizzenbuch“ [Manuskriptsammlung]. BA, Nachlass Ludwig von Ficker, Sign. 81/14-1, sowie, in geringfügig anderer Ausführung, Sign. 81/21-1.

31. Ebd.

32. Ebd.

33. Vgl. Karlheinz Rossbacher, „Heimatkunst der frühen Moderne“, in York-Gothart Mix (Hg.), Naturalismus, Fin de Siècle, Expressionismus 1890-1918 (= Hansers Sozialgeschichte der deutschen Literatur, Band 7), München, Wien, Carl Hanser Verlag, 2000, S. 300-313. 
Dynamik [...] versuchen ihre Autorexistenz zu legitimieren, indem sich dem Innovationszwang unterwerfen. ${ }^{\text {“34 }}$

\section{Sündenkinder und Christnachtstragödien}

Für Fickers Dramenproduktion ist zu konstatierten, dass er, bezogen auf die theoretische Programmatik des Naturalismus, nur bedingt in dieselbe thematische Breite wie ihm nahestehenden Dichterkollegen eintauchte. In seinen Stücken finden sich die Motive „Verlogenheit der Gesellschaft - Verhältnis zur Frau - Sexualität und Liebe - Erziehung - Religion - Armut, Elend und Krankheit“ ${ }^{\text {"35 }}$ lediglich in Ansätzen behandelt und nicht in derselben Radikalität umgesetzt, wie es beispielsweise bei Schamann der Fall ist.

Sündenkinder war das erste Drama, das Ficker in der kurz zuvor neu gegründeten Österreichischen Verlagsanstalt Linz veröffentlichte. Ficker hatte das Dramenmanuskript bereits im Winter 1898 beendet, wie ein Brief Carl Spitzwegs belegt ${ }^{36}$; die Uraufführung fand schließlich am 13. Jänner 1900 mit einigem Erfolg im Innsbrucker Stadttheater statt. ${ }^{37}$ Der Zweiakter beschränkt sich auf ein Repertoire von lediglich fünf Figuren, wie er auch vom Handlungsverlauf recht simpel gehalten ist. Das Stück thematisiert das gesellschaftliche Problemfeld der unehelichen Kinder und damit verbundene Fragen nach der Ehe und der Ehre. Die Hauptfiguren sind der Student Philipp, der bei seinem Onkel wohnt, und Margreth, die für den Onkel den Haushalt besorgt. Philipp und Margreth sind liiert und die Ehe scheint beschlossene Sache. Unvermittelt taucht jedoch der reiche Fabrikantensohn Felix Wartenberg auf, eine frühere Bekanntschaft Margreths, der sie aber nach

34. Klaus-Michael Bogdal, Historische Diskursanalyse der Literatur. Theorie, Arbeitsfelder, Analysen, Vermittlung, Opladen, Westdeutscher Verlag, 1999, S. 155. Ein Theaterzettel aus dem Jahr 1900 demonstriert diese Ambivalenz. Ficker hatte am 26. Mai als Schauspielleiter eine Aufführung von Hermann Sudermanns Einakter „Fritzchen“ inszeniert. Im Rahmen der Wohltätigkeitsveranstaltung, in der die Aufführung stattfand, präsentierte er zeitgleich, gemeinsam mit Carl Spitzweg, „einige Szenen aus Göthes Faust“; vgl. die Abb. in Unterkircher, „Ludwig von Ficker", a.a.O., S. 34.

35. Reinhild Kaufmann, „Franz Schamann und Ludwig von Ficker“, in Walter Methlagl u.a., Untersuchungen zum, Brenner, a.a.O., S. 177-185; hier S. 178.

36. Brief Carl Spitzweg an Ludwig von Ficker, 22.12.1898. BA, Nachlass Ludwig von Ficker, Sign. 42/7-4.

37. Unterkircher, „Ludwig von Ficker“, a.a.O., S. 36. 
einer Schwangerschaft und dem Tod des Kindes verlassen hatte, und ihre Verfehlung wird ruchbar. Philipp kann Margreth wegen der subjektiv empfundenen Ehrverletzung nicht verzeihen. Er löst die Verlobung, obwohl zwischenzeitlich klar wird, dass auch er ein uneheliches Kind ist, und fordert, trotz der Vermittlung des Onkels, Wartenberg zum Duell. Der Ausgang desselben bleibt unbestimmt, da er bereits außerhalb der Dramenhandlung liegt.

Ein provokanter Titel wie „Sündenkinder“ ist durchaus dafür geeignet, eine sozialkritische Komponente bzw. Kritik an gesellschaftlichen Realitäten zu transportieren. ${ }^{38}$ Die Umsetzung des kritischen Moments gelang Ficker aber nicht in jenem Maße, wie es andere Naturalisten - so auch Hermann Sudermann mit seinem Stück Ehre (1889), an dem sich Ficker zweifelsohne orientiert hat - in ihren Sozialdramen schafften. Wohl erhält der soziale Konflikt zwischen Reich und Arm im Drama handlungsrelevante Funktion; allerdings wird er von Ficker nicht im Geiste naturalistischer Radikalität als „die ewige Menschheitsfrage“39 präsentiert und interpretiert, sondern vielmehr reichlich plakativ konstruiert und gleichzeitig auf fast schon schwülstige Weise gebrochen, als die Figur der Margreth reüssiert: „Soll uns versagt sein, was jeden Reichen glücklich macht, nur weil wir arm sind, bitter arm? Zum Leben sind auch wir geboren. Und was ist Leben ohne Liebe?" 40

1901 erschien, abermals in der Österreichischen Verlagsanstalt, das zweite Drama aus Fickers Feder unter dem Titel Und Friede den Menschen! Eine Christnachtstragödie ${ }^{41}$. Ficker stellte dem Dramentext - gewissermaßen als programmatische Widmung - eine Strophe aus einem Gedicht von Otto Erich Hartleben voran, in der es heißt: „Und ob ihr Klugen auch mein Wollen höhnt - / Und ob ihr Frommen mich entsetzt verpönt - / Und ob ihr Zarten meine Worte flieht - / Hart ist das Leben, hart sei auch mein Lied!" 42 Schon in diesem Zitat liegt der wesentliche Unterschied zu den "Sündenkindern“ und damit eine

38. Der Titel musste auf das Innsbrucker Publikum tatsächlich anstößig gewirkt haben; vgl. Neue Tiroler Stimmen, 15.01.1900: „Das Logenpublicum war in seiner Mehrheit, offenbar durch den vielversprechenden Titel abgeschreckt, der Vorstellung fern geblieben."

39. Michael Georg Conrad, „Flammen! Für freie Geister“, in Theo Meyer (Hg.), Theorie des Naturalismus, Stuttgart, Reclam, 1997, S. 56-59; hier S. 58.

40. Ficker, Sündenkinder, S. 71.

41. Und Friede den Menschen! Eine Christnachtstragödie von Ludwig v. Ficker, Linz u.a., Oesterreichische Verlagsanstalt, 1901.

42. Ebd., Klappentext. 
stärkere Annäherung an die Positionen des Naturalismus begründet, denn Hartlebens Verse nehmen bereits den Kern der dramatischen Handlung und damit auch die Form von gesellschaftlicher Kritik, die Ficker in das Stück zu packen versuchte, vorweg; es sind insbesondere die Aspekte der „Frömmigkeit“ und der „Härte“, die im Stück den für die Hauptfigur tragischen Ausgang bestimmen.

Das Drama thematisiert (in motivischer Anlehnung an Max Halbes Jugend) erneut den gesellschaftlichen Umgang mit illegitimen Kindern und den damit verbundenen Ehrbegriff. Die Hauptfigur ist Gusti Webersink, eine junge Wöchnerin, deren Verlobter kurz zuvor bei einem Kirchenbrand ums Leben gekommen ist. Weil sie aufgrund der Aufregung einen Zusammenbruch erlitten hat und sich dem Tod nahe fühlt, wird nach dem Pfarrer Wendt geschickt, der sie mit den Sterbesakramenten versehen soll. Zwar konstatiert der hinzugezogene Arzt, dass die Frau auf dem Weg der Besserung sei, doch das Drama gelangt an einen Wendepunkt, als der Pfarrer von Gusti Abbitte für „den schweren Frevel an der Heiligkeit des Leibes" ${ }^{43}$, den sie begangen habe, verlangt. Eine solche will sie aber nicht leisten, woraufhin Wendt ihr das Sakrament verweigert und Gusti stirbt, ohne die Absolution erhalten zu haben. Der Pfarrer wird von den Dorfbewohnern vertrieben, der Dorflehrer nimmt sich des Kindes an.

Die im Vergleich zu den Sündenkindern veränderte Herangehensweise an den Stoff macht deutlich, auf welche Weise Ficker versucht hat, sich jenen Forderungen, die Hugo Greinz in seinem Appell für eine Provinzliteratur aufgestellt hatte, in der Praxis anzunähern. Die Sprache der Figuren ist stärker dialektal gefärbt, das Figurenrepertoire wurde insgesamt erweitert und erscheint stärker stratifiziert. Die Handlung ist ebenfalls nicht mehr im städtischen Milieu verortet, sondern „in einem grösseren Gebirgsdorf des tirolisch-deutschen Grenzbezirks" 44 . Insofern verschiebt sich auch der Fokus auf die naturalistisch motivierte Schilderung einer dörflichen Lebensrealität, wenngleich im dramatischen Konflikt keine deterministische Letztbegründung auszumachen ist (vor allem deshalb, weil Ficker trotz der Kritik an der Institution Klerus die metaphysische Dimension weitgehend unhinterfragt lässt).

Anders als die Sündenkinder gelangte Und Friede den Menschen! nie zur Aufführung, wie sich auch die Rezeption eher verhalten gestaltete; es existieren insgesamt nur wenige Besprechungen, die nicht nur positiv

43. Ebd., S. 65.

44. Ebd., S. 7. 
ausfielen. Hans Sittenberger rezensierte das Drama in Das litterarische Echo :

[...] Im wesentlichen handelt es sich immerhin um Zustandsschilderung ganz im Sinne des Naturalismus. Nicht alles ist da gelungen. Allzu oft bleibt Ficker in Deklamation befangen, auch ist er keineswegs selbständig. Es ist wunderlich zu sehen, wie ihm Anzengruber und Hauptmann in friedlichem Vereine zu Paten stehen. Aber die fremden Elemente fließen doch zu einer Einheit zusammen. Besonders gut versteht sich Ficker darauf, Stimmungen zu erwecken, sie machtvoll zu steigern und ausklingen zu lassen. Vor allem aber hat er Blicke in Menschenherzen gethan, und manch ein Wort giebt uns Kunde von dem, was er heimlich gesehen. Das ist ein Zeichen echter Begabung, und so viel an seiner Christnachtstragödie stört, so wenig sie selbst noch Erfüllung ist, als eine Verheißung mag sie willkommen sein. ${ }^{45}$

Trotz des wohlwollenden Tons der Rezension sollte sich Fickers „echte Begabung" nicht auf dem Gebiet der Dramenproduktion, sondern erst eine Dekade später in der Funktion des Zeitungsherausgebers und Kulturvermittlers erfüllen. Die nicht zu leugnenden kompositorischen Schwächen stellten einen Faktor dar, der einer breiteren Aufnahme des Stückes über den lokalen Raum hinaus entgegenstand. Letztlich führten aber der gegen die Institution der katholischen Kirche gerichtete Impetus und der - zumindest latent vorhandene - sozialkritische Unterton, der in Und Friede den Menschen! vor allem aufgrund der stofflichen Annäherung an die Regionalliteratur etwas stärker zum Ausdruck kommt als in den Sündenkindern, zu einer Rezeption, die das Stück als dezidiert naturalistisch wahrzunehmen begann. Eine (allerdings recht späte) Würdigung stellt hier das Urteil Sigfrid Hoeferts dar, der konstatierte, dass Ficker mit dem Stück „dem Naturalismus entschieden gehuldigt “46 habe.

\section{Vom Föhn zum Brenner}

In den Jahren zwischen 1904 und 1909 ruhte die Tätigkeit Fickers auf künstlerischem Gebiet. Er studierte stattdessen, unternahm längere Reisen und hielt sich unter anderem in Paris, Rom und Siena auf. ${ }^{47}$

45. Hans Sittenberger, „Oesterreichische Dialektstücke“, Das litterarische Echo. Halbmonatsschrift für Litteraturfreunde, Vierter Jahrgang, H. 8, Januar 1902, Sp. 538-539.

46. Sigfrid Hoefert, Das Drama des Naturalismus, Stuttgart, Metzler, 1993, S. 95.

47. Vgl. Unterkircher, „Ludwig von Ficker“, a.a.O., S. 4 O. 
Im Frühjahr 1909 kam es zu einer Reaktivierung, denn Ficker wurde, auf Initiative von Eberhard Weittenhiller, vom Herausgebergremium der unter dem Banner der „Jung-Tirol“-Bewegung firmierenden, neu gegründeten Kulturzeitschrift Föhn zur Mitarbeit bewogen. Doch schon nach wenigen Wochen kam es zu Streitigkeiten bezüglich der Kompetenzverteilung und des Leitungsanspruchs. Im Frühherbst 1909 hatte sich das Missverhältnis zu den Föhn-Verantwortlichen Rudolf Brix, Franz Kranewitter und insbesondere zu Richard Wilhelm Polifka bereits $\mathrm{zu}$ einem handfesten Zerwürfnis gesteigert. Angesichts dieser Situation reifte der Plan zur Gründung einer eigenen Zeitschrift.

Am 13. September 1909 wurde Ficker vom Wiener Autor Robert Michel in einem Brief auf einen Föhn-Beitrag zur Jubiläums-Kunstausstellung ${ }^{48}$, die in Innsbruck seit 21. Juli anlässlich der 100-Jahr-Feier der Erhebung Tirols gegen die napoleonische Herrschaft veranstaltet wurde, aufmerksam gemacht. Eine Passage des Artikels erregte dabei Fickers Gemüt in besonderer Weise, weil darin zu den Epochenbegriffen „Naturalismus“ und „Moderne“ Stellung genommen worden war. ${ }^{49}$ Ficker antwortete:

Was soll das heißen, daß Bahr mit der ,Moderne' den ,Naturalismus' überwand?! Der Naturalismus ist doch im Gegentheil die große Revolution, die die Moderne begründet hat. Und was hat Bahr damit zu schaffen gehabt? Das „Schlagworteprägen“ ist gewiß ein recht secundäres Verdienst..$^{50}$

Ficker gab mit dieser kurzen Briefstelle - der einzigen im (publizierten) Briefwechsel, in der er expressis verbis auf den Begriff des „Naturalismus“ zu sprechen kommt - zumindest im Ansatz einen Blick auf sein Verständnis von literarhistorischen Zusammenhängen frei. Dem Brief war ein (verloren gegangenes) Schreiben Michels vorangegangen, der darin, wie sich aus dem Antwortbrief Fickers rekonstruieren lässt, die Vermutung angestellt hatte, dass der anonyme Föhn-Artikel über

48. Vgl. Allgemeiner Tiroler Anzeiger, 26.07.1909.

49. Die inkriminierte Stelle im Föhn, auf die sich Michel in seinem Brief bezogen hatte, lautet wie folgt: „Täuschen wir uns nicht selbst: Auch wir, die mitten drin waren im froh-kräftigen Streite und mit jungem Drauflosgehertum mitkämpfen halfen, als Bahr Schlagworte prägte und mit der ,Moderne' den ,Naturalismus' überwand, - wir alle sind im Herzen doch nicht ganz frei geworden von Ueberlieferung und Grundsatz, die man uns auf den Schulbänken eingetrichtert hat." Anonym, „Betrachtungen zur Kunstausstellung", in Der Föhn, 1. Jg, 1909, S. 97-103; hier S. 97.

50. Brief Ludwig von Ficker an Robert Michel, 13.09.1909, in Ludwig von Ficker, Briefwechsel 1909-1914, hg. von Ignaz Zangerle, Walter Methlagl, Franz Seyr, Anton Unterkircher, Salzburg, Otto Müller Verlag, 1986, S. 11-13; hier S. 12. 
die Kunstausstellung von Ficker verfasst worden war, was dieser aber vehement verneinte und mit der Polemik gegen Bahr konterte. Die Kritik erstreckte sich allerdings nur vordergründig auf den kulturpolitischen Opportunismus Bahrs.

Ficker interpretierte das Diktum von der „Überwindung des Naturalismus" ex post im Abstand von knapp zwanzig Jahren aus dem Zeithorizont des anbrechenden expressionistischen Jahrzehnts aus und glaubte, die zeitgenössische „Moderne“ und ihre Genealogie aus einem völlig anderen Blickwinkel zu sehen, als es Bahr getan hatte. Tatsächlich unterscheidet sich seine Aussage inhaltlich aber kaum von den Überlegungen Bahrs, dass der Naturalismus zur „Entbindung der Moderne“ 51 beigetragen hätte. Diese Koinzidenz lässt den Schluss zu, dass Ficker Bahrs Text nicht gekannt hat; von zentraler Bedeutung aber ist die Tatsache, dass er den Naturalismus nicht als Fortsetzung bzw. Radikalisierung des Realismus, sondern als „Revolution“ im Sinne eines Einschnitts bzw. einer Neukonstitution wahrgenommen hat.

Mit seiner Abwehrhaltung verdeutlichte Ficker zudem gegenüber Michel, der über Leopold von Andrian Kontakt zum "Jung-Wien“ unterhielt, seine Distanz zur Wiener "Nervenkunst“ und versuchte gleichzeitig, seinen eigenen Standpunkt zu untermauern bzw. seine Pläne für eine neue Zeitschrift im Westen der Monarchie zu legitimieren. Er sparte deshalb nicht mit Kritik an den Wiener Autoren, die der neuen Subjektivität anhingen (namentlich Robert Müller mit dem „Ruf“), und der Qualität ihrer Literatur:

Das sind unsere jungen Nerven-,Heroiker'. Daß Gott erbarm'! Unsere Volldampf-Hysteriker! Hol sie der Teufel! Ihre Hingegebenheit an das Leben ist besinnungslose Schweinerei. Und wenn sie den Hermann Bahr feiern, feiern sie ihn mit Recht. Es stimmt alles so wunderbar, und Unehrlichkeit ist bei ihnen Ausdruck höchster Ehrlichkeit. So ,begabt' sind sie! $!^{52}$

Auf der Grundlage der Abwendung sowohl von der Wiener Moderne als auch vom Tiroler Regionalismus veröffentlichte Ficker ab Juni 1910 den Brenner. Vier Jahrzehnte später blickte er, am Grabe seines ersten Hauptmitarbeiters Carl Dallago stehend, auf die „Übergangszeit um die Jahrhundertwende mit ihrem Einbruch stürmischer Bewegungen in die

51. Hermann Bahr, Die Überwindung des Naturalismus. Als zweite Reihe von ,Zur Kritik der Moderne', Dresden-Leipzig, Pierson, 1891, S. 155.

52. Brief Ludwig von Ficker an Ludwig Erik Tesar, 24.10.1913, in Ludwig von Ficker, Briefwechsel 1909-1914, a.a.O., S. 185-186; hier S. 186. 
Stagnation des Althergebrachten " 53 zurück. Er spielte damit auf die hier skizzierte Phase der persönlichen wie institutionellen Konsolidierung an, aus der schließlich mit dem Brenner sein Lebensunternehmen erwuchs. Die „Übergangszeit“ äußerte sich, auf die ersten Nummern des Brenner bezogen, insbesondere in einer inhaltlichen Mehrdimensionalität, denn jene kulturelle Entität, die Ficker im Brief an Robert Michel mit dem Begriff der Moderne umrissen und gegenüber dem Naturalismus und der Wiener "Nervenkunst“ abgegrenzt hatte, war freilich auch im Brenner nicht konkret definiert und deshalb auch in den Beiträgen nur schwer greifbar. Schon die Mitarbeit Dallagos am Brenner, dessen kulturkritische Positionen nach der Sezession vom Föhn ein Kontrastprogramm zum revolutionären Duktus der „Jung-Tiroler" darstellen sollte, bewirkte durch die Forderung nach Apperzeption des Naturmenschentums bzw. der Ablehnung des von ihm als "philiströs“ gebrandmarkten Zivilisatorischen, wie es das (Bildungs-)Bürgertum verkörperte, eine Bewegung weg von der Richtung, die den Föhn noch an die Provinzliteratur bzw. die Heimatkunstbewegung angebunden hatte. Ficker rekurrierte ein letztes Mal auf den Naturalismus, als er im Brenner Auszüge aus Schamanns Texten veröffentlichte, ${ }^{54}$ doch schon unmittelbar danach verebbte im die Beschäftigung mit Autoren, die dem Naturalismus zumindest nahestanden. Abseits der öffentlichen Agitation setzten sich Dallago und Ficker allenfalls noch brieflich mit Ibsen auseinander, spätestens mit der Begegnung Fickers mit Georg Trakl schwand auch dieses Thema.

Summarisch kann festgestellt werden, dass weniger die literarische Qualität von Fickers Texten ausschlaggebend war - diese lässt sich getrost als bestenfalls mittelmäßig bezeichnen -, sondern vielmehr der Status Quo des jungen Autors, der sich in einer Situation behauptete, die durch diskursive Auseinandersetzungen insbesondere auch im künstlerischen Bereich geprägt war. Er sah sich mit einer Vielfalt von Einflüssen

53. Ludwig von Ficker, „Frühlicht über den Gräbern. [Zur Geschichte des „Brenner“] 1949-1954. I: Am Grabe Carl Dallagos“, in ders., Denkzettel und Danksagungen, München, Kösel Verlag, 1967, S. 185-198; hier S. 185.

54. Franz Schamann, „Der Indra“, in Der Brenner, I. Jahr, 1. Halbb., 15.06.1910, S. 32-39, sowie die Fortsetzung u.d.T. „Adolf“, in ebd., I. Jahr, 1. Halbb., H. 3, 01.07.1910, S. 59-66; ders., „Habsburg. Ein szenischer Prolog“, in ebd., I. Jahr, 1. Halbb., H. 6, 15.08.1910, S. 128-138, sowie ebd., I. Jahr, 1. Halbb., H. 7, 01.09.1910, S. $167-174$. 
konfrontiert, die durch ein komplexes gesellschaftliches Dispositiv erzeugt wurden, das in seinem Fall durch den kulturellen Transfer um eine zusätzliche Dimension erweitert wurde. Der Briefwechsel aus dem Ende des 19. Jahrhunderts bis zur Gründung des Brenners liefert Einblicke in die Verfahrensweisen und Praktiken, mit denen ein Angehöriger einer jungen Dichtergeneration auf dem kulturellen Feld einen Kampf um den „Willen zum Wissen“ 55 ausfocht, der nur unzureichend mit der Dichotomie von „Alt-Tirol“ versus „Jung-Tirol“ beschrieben ist, sondern vielmehr das programmatische Spannungsfeld der Moderne reflektiert. Im konkreten Fall fügt sich die Frage, ob ein Einfluss des Naturalismus auf sein Schaffen festzustellen ist, deshalb in die Antwort, die Werner Michler bereits auf allgemeiner Ebene für den Naturalismus österreichischer Prägung gegeben hat: „das kommt darauf an. “56

Die literarhistorische Bewertung der schriftstellerischen Leistung Ludwig von Fickers mag im Sinne kanonischer Maßstäbe nachrangig sein. Der Einfluss, den er - zumindest ab dem Zeitpunkt, an dem der Brenner die kulturelle Bühne betreten hat -, weitgehend aus dem Verborgenen heraus auf die europäische Kultur ausgeübt hat, steht hingegen außer Frage. Auch wenn Ficker später nicht mehr über seine frühen schriftstellerischen Versuche sprechen wollte, bleibt doch die Tatsache bestehen, dass er in der Zeit des kulturellen Umbruchs um die Jahrhundertwende eine Form der kunsttheoretischen Sozialisation vollzog, die als Initialzündung für seine spätere Tätigkeit als Kulturvermittler angesehen werden kann.

55. Michel Foucault, Die Ordnung des Diskurses, Frankfurt am Main, Fischer, 1973, S. 14.

56. Werner Michler, „Zur Frage eines österreichischen Naturalismus“, in Roland Innerhofer, Daniela Strigl (Hg.), Sonderweg in Schwarzgelb? Auf der Suche nach einem österreichischen Naturalismus in der Literatur, Innsbruck, Studienverlag, 2016, S. 21-32; hier S. 21. 